patients, concurrent renal biopsy was performed, unless contraindicated. The ISN/RPS criteria were used to assess the histopathologic features of LN. Those Patients were further subcategorized into 2 groups; active proliferative (ISN/RPS classes III/IV) and non-proliferative (classes I/II/V).

Results: The serum concentrations of $\mathrm{Axl}$ and ferritin were significantly higher in patients with active SLE than inactive SLE $(3765 \pm 235$ vs. $2513 \pm 130 \mathrm{pg} / \mathrm{ml}, P=$ $0.001)$ and $(111 \pm 26$ vs. $18 \pm 4 \mathrm{ng} / \mathrm{ml}, P=0.0001)$ respectively. Serum Axl levels were significantly higher in active renal versus active non-renal SLE patients $(3765 \pm 235.3$ vs. $2825 \pm 200.7 \mathrm{pg} / \mathrm{ml}, P=0.04)$. In the active renal patients with paired kidney tissue and blood samples, none of the biomarkers tested discriminated classes of LN, although serum Axl, ferritin and IGBPB4 levels were higher in the proliferative subgroup. The levels of Axl, ferritin and IGFBP4 correlated significantly with SLEDAI scores (Axl, r= 0.58, $P<0.0001$; ferritin, $\mathrm{r}=0.53, P<0.0001$; IGFBP4, $\mathrm{r}=0.229, P=$ 0.03). However, only serum Axl levels correlated significantly with the renal SLEDAI $(\mathrm{r}=0.46, P=0.01)$. The levels of Axl, IFGBP4 and sTNFR2 correlated with decreased C3 levels $(r=-0.54, P<0.0001 ; r=-0.29, P=0.007 ; r=-0.29, P=0.007)$ respectively. Only serum Axl and ferritin correlated with urinary PCR $(r=0.42, P<0.0001$; $r=$ $0.22, P=0.04$ ) respectively. These markers were more specific, but less sensitive, in detecting concurrent SLE activity than elevated anti-dsDNA or decreased C3. The specificity values of serum ferritin and IGFBP4 for concurrent active lupus nephritis were higher than anti-dsDNA or C3. Serum ferritin was the best predictor of global SLE activity (AUC 0.81, $P<0.0001$ ), followed by C3 (AUC 0.79, $P<0.0001$ ) then Axl (AUC 0.71, $P=0.002$ ), while both $A x l$ and $C 3$ were the best predictors of lupus nephritis activity (AUC 0.72 , both).

Conclusion: In pediatric SLE patients, serum ferritin and Axl perform better than traditional yardsticks in identifying disease activity, either global or renal. The performance of these serum markers should be explored further in a longitudinal cohort of pediatric SLE patients.

References:

[1] Wu T, Ding H, Han J, et al. Antibody-Array-Based Proteomic Screening of Serum Markers in Systemic Lupus Erythematosus: A Discovery Study. J Proteome Res. 2016 Jul 1;15 (7): 2102-14.

Disclosure of Interests: None declared

DOI: 10.1136/annrheumdis-2020-eular.1581

\section{SAT0235 \\ THE EFFECT OF ANTIPHOSPHOLIPID ANTIBODIES ON APTT WAVEFORM PATTERNS}

Y. Suzuki ${ }^{1}$, A. Mitsui ${ }^{1}$, Y. Yamamoto ${ }^{1}$, K. Noda ${ }^{1}$, A. Nakajima ${ }^{1} .{ }^{1}$ Mie University Hospital, Center for Rheumatic Diseases, Tsu,Mie Prefecture, Japan

Background: Patients with antiphospholipid antibody (aPL) are said to be at increased risk for thrombosis, however it is difficult to predict whether they will develop thrombosis. In recent years, it has been revealed that the characteristics of the second derivative curve of APTT waveform with aPL positive patient is biphasic changes ${ }^{1,2}$. As first step in predicting the risk of thrombosis, we sought to understand the effect of aPL on APTT waveform patterns.

Objectives: To analyze the characteristics of APTT waveforms according to the background diseases and the presence of aPL

Methods: Patients who underwent coagulation function tests from 2017 to 2019 were analyzed. A coagulation waveform (Clot waveform: CW) was drawn using a fully automatic coagulation time measuring device manufactured by Instrumentation Laboratory From the APTT waveform, the 1st derivative curve (DC) indicating the coagulation speed and the $2 \mathrm{nd}$ DC indicating the coagulation acceleration were depicted to measure the 1st DC height, 2nd DC peak 1 time, and 2nd DC peak 1 height $\left(\right.$ Figure ${ }^{2}$ ). Patients were devided into CTD with aPL-negative patients (group A), aPL-positive patients with no prior thrombosis (group B), and antiphospholipid antibody syndrome (APS) (group C). Patients characteristics and aPL (anti-cardiolipin [CL] antibody IgM, anti-CL antibody IgG, anti-CL 32 GP1 complex antibody, LA-APTT, and LA- DRVVT) status were examind. A further analysis was performed according to the numbers of positive aPL. Comparison between the three groups were made by the one-way ANOVA method, with significant differences set as $p$-values $<0.1$. Factors with significant differences were analyzed by Steel-Dwass test. APTT waveforms was analyzed according to the numbers of positive aPL by least squares methods. Furthermore, to determine the cut off value of APTT, 1st DC height, 2nd DC peak 1 time, and 2nd DC peak 1 height for each case with 2 or more positive aPLs and 3 positive aPLs, area under the curve (AUC) of the receiver operating characteristic (ROC) curve, sensitivity and specificity were caliculated.

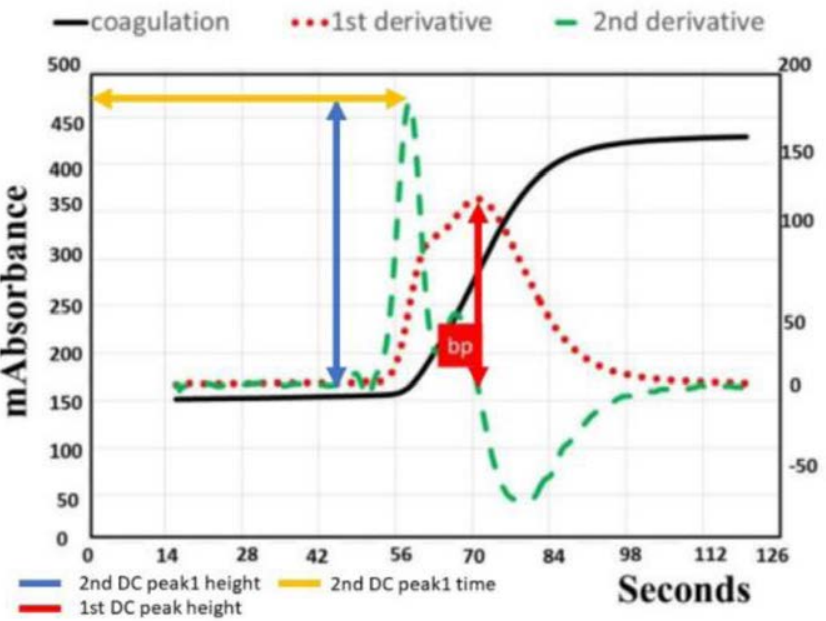

Figure 1.

Results: The APTT waveform was analyzed in 61 patients (51 women, 83.6\%) with average age of $54.1 \pm 17.1$ years. Group A was 26 cases, Group B was 18 cases, and Group C was 17 cases. APTT, 2nd DC peak1 time, 2nd DC peak1 height, 1st DC peak time were significantly different among $A, B$, and $C$ groups ( $p<0.01$ ). APTT, 1st DC peak height, 2nd DC peak 1 time, and 2nd DC peak 1 height differed among the number of $\mathrm{aPL}$ ( $p<0.01$, respectively). APTT and 2 nd DC peak1 time prolonged by 9.43 (seconds) and 16.3 (seconds) respectively according to the number of aPLs increased, and 1st DC peak height (mabs/s) and 2nd DC peak1 height $\left(\mathrm{mabs} / \mathrm{s}^{2}\right)$ decreased by 56.4 (mabs/s) and 223.9 $\left(\mathrm{mabs} / \mathrm{s}^{2}\right.$ ) respectively according to the number of aPLs decreased (Table 1). APTT> 35.2 (seconds) (sensitivity $80 \%$, specificity $80.4 \%$ ), 2nd DC peak1 height> 302 (mabs $/ \mathrm{s}^{2}$ ) (sensitivity $80 \%$, specificity $91.3 \%$ ) were relevant to the presence of two or more aPLs and APTT> 35.2 (seconds) (sensitivity 100\%, specificity $80 \%)$, 2nd DC peak1 height> $302\left(\mathrm{mabs} / \mathrm{s}^{2}\right)$ (sensitivity $100 \%$, specificity $90 \%$ ) were relevant to the presence of three aPLs.

Table 1.

\begin{tabular}{llllll}
\hline The number of positive aPL & 0 & 1 & 2 & 3 & $p$ value \\
\hline The number of cases & 27 & 19 & 4 & 11 & \\
APTT & 28.9 & 30.9 & 31.1 & 60.7 & 0.0001 \\
(seconds) & {$[26.8,31.4]$} & {$[29.1,38.2]$} & {$[27.3,54.2]$} & {$[45.9,73.7]$} & \\
2nd DC peak time (seconds) & 29.2 & 33.7 & 36.1 & 75.8 & 0.0001 \\
& {$[26.8,30.7]$} & {$[31,41.7]$} & {$[31.5,99]$} & {$[50.5,102.4]$} & \\
2nd DC peak height (mabs/s $)$ & 839.9 & 669.6 & 608.4 & 119.3 & 0.0001 \\
& {$[666.1,962.2][346.4,946][137.8,956.7][30.6,196]$} & \\
1st DC peak height (mabs/s) & 309.6 & 271.5 & 241.8 & 135.6 & 0.0001 \\
& {$[260.6,355.1][168,353]$} & {$[96.3,364.0]$} & {$[76.8,163]$} & \\
\hline
\end{tabular}

Conclusion: The presence of aPL was more related to the 2nd DC peak1 height of APTT waveform than APTT. A detailed review of the APTT waveform may further predict future thrombosis risk.

References:

[1] Tokunaga N, et al. Blood Coagul Fibrinolysis. 2016;27:474-476.

[2] Matsumoto T, et al. Int J Hematol. 2017;105:174-183.

Disclosure of Interests: YASUO SUZUKI: None declared, Asako Mitsui: None declared, Yoshiki Yamamoto: None declared, Kentaro Noda: None declared, Ayako Nakajima Grant/research support from: AN has received research grants from Chugai Pharmaceutical Co., Ltd., Mitsubishi Tanabe Pharma Co., Pfizer Japan Inc., Consultant of: AN has consultant fee from Nippon Kayaku Co. Ltd., Speakers bureau: AN has received speaker's fee from AbbVie Japan GK, Actelion Pharmaceuticals Japan LTD., Asahi Kasei Pharma Co., Astellas Pharma Inc., Ayumi Pharmaceutical Co., Bristol Myers Squibb Co., Ltd., Chugai Pharmaceutical Co., Ltd., Eisai Co., Ltd., Eli Lilly Japan K.K., GlaxoSmithKline K.K., Hisamitsu Pharmaceutical Co. Inc., Kyorin Pharmaceutical Co. Ltd., Mitsubishi Tanabe Pharma Co., Otsuka Pharmaceutical Co. Ltd., Pfizer Japan Inc., and Teijin Pharma Ltd.

DOI: 10.1136/annrheumdis-2020-eular.1833 